Wayne M. Tsuang, MD, MHS

Department of Pulmonary Medicine, Respiratory Institute,

Cleveland Clinic
Marie M. Budev, DO, MPH

Department of Pulmonary Medicine, Respiratory Institute,

Cleveland Clinic

\title{
COVID-19 and lung transplant patients
}

\section{Posted April 9, 2020}

\section{ABSTRACT}

COVID-19 is a novel respiratory disease leading to high rates of acute respiratory failure requiring hospital admission. It is unclear if specific patient populations such as lung transplant patients are at higher risk for COVID-19. Some reports suggest that transplant patients may not be at higher risk if proper social distancing and preventive measures are employed. Efforts to ensure the safety of wait-listed patients, transplant recipients, and healthcare workers are underway. Recommendations for the care of lung transplant patients during the COVID-19 pandemic are discussed and will likely change as the pandemic evolves.

\section{INTRODUCTION}

COVID-19 is a novel respiratory disease leading to high rates of acute respiratory failure requiring hospital admission. ${ }^{1}$ It is still unclear if specific patient populations such as lung transplant patients are at higher risk for COVID-19. Published reports suggest that transplant patients may not be at higher risk if proper social distancing and preventive measures are employed. ${ }^{2}$ Transplant centers, in coordination with several national transplant societies, ${ }^{3}$ are rapidly assessing and responding to the pandemic to ensure the safety of wait-listed patients, transplant recipients, and healthcare workers and to facilitate effective stewardship of available hospital resources. Current recommendations for the care of lung transplant patients during the COVID-19 pandemic are summarized below. It is important to note that these recommendations may change as the pandemic evolves.

The statements and opinions expressed in COVID-19 Curbside Consults are based on experience and the available literature as of the date posted. While we try to regularly update this content, any offered recommendations cannot be substituted for the clinical judgment of clinicians caring for individual patients.

doi:10.3949/ccjm.87a.ccc004

\section{A PATIENT WHO HAD A LUNG TRANSPLANT CALLS WITH SYMPTOMS OF A RESPIRATORY INFECTION. WHAT SHOULD I DO?}

The recommendation is to have a low threshold for COVID-19 testing in a patient with mild symptoms and also to concomitantly test for other respiratory viruses such as influenza or respiratory syncytial virus. Atypical presentations of COVID-19 infection include the absence of fever and should be considered in a transplant patient. Local policies and resources will dictate whether COVID-19 testing should be done in the asymptomatic transplant patient. We also recommend notifying the patient's transplant center as soon as possible for additional guidance on clinical management.

Most transplant centers currently test for COVID19 infection using polymerase chain reaction on nasopharyngeal or oropharyngeal swabs, similar to the testing protocol used in the general population. Patients with mild symptoms and no shortness of breath or hypoxia should be quarantined at home for 2 weeks and monitored frequently for these symptoms via phone or telehealth visits. Those with signs of moderate symptoms including shortness of breath or hypoxia should be directed to the nearest emergency room and admitted for supportive care and consideration for COVID-19-specific care protocols.

\section{A PATIENT WHO HAD A LUNG TRANSPLANT IS ADMITTED TO THE HOSPITAL WITH HYPOXIA FROM COVID-19. WHAT SHOULD I DO?}

When a patient with COVID-19 and moderate to severe symptoms is admitted, it is important to recognize he or she may rapidly deteriorate. Early endotracheal intubation should be considered, and noninvasive positive pressure ventilation should be limited to avoid spread via aerosolization. However, individual center practices and resources will dictate intubation timing. Concomitant antimicrobial or antifungal therapy may be needed.

Current guidelines recommend holding mycophenolate mofetil or azathioprine in patients with mod- 
erate or severe disease and closely monitoring them for possible graft rejection. In the severely ill, who may have profound refractory hypoxemia or hypercapnia, extracorporeal membrane oxygenation may be needed in addition to invasive ventilation.

Transplant centers are tracking their patients who are positive for COVID-19 and are collecting data to report to registries. This will allow us to identify important clinical trends that can improve how we treat lung transplant patients. Thus, we encourage providers to update the patient's transplant team so the necessary clinical data can be forwarded to these registries. Until we have more information specific to lung transplant patients, we recommend using local best practices to manage immunosuppressed patients with acute respiratory failure. There are ongoing national discussions on the development of COVID-19-specific therapies. A history of lung transplant should not preclude a patient from consideration for medications or trials.

\section{WHAT KIND OF MEDICATIONS DO LUNG TRANSPLANT PATIENTS USUALLY TAKE?}

There are two general types of lung transplant medication regimens: immunosuppression and prophylaxis. The doses differ for each patient depending on the transplant center and how recently the transplant was performed.

For immunosuppression, patients may take up to 3 different medications. The first is a calcineurin inhibitor, commonly tacrolimus and sometimes cyclosporine. These medications are usually taken twice a day orally, and tacrolimus has a sublingual option if a patient has no oral access. Blood levels are often checked to assess for a therapeutic drug level, and the target range will vary depending on the transplant center. Next, patients might be on an antimetabolite such as mycophenolate or azathioprine, and finally an oral steroid such as prednisone. For prophylaxis, three broad medication areas are typically covered: Pneumocystis prevention, fungal prevention (usually targeting Aspergillus), and cytomegalovirus prevention. We recommend contacting the patient's transplant center because the medication, duration, and doses in a lung transplant patient will vary.

\section{A PATIENT WITH END-STAGE LUNG DISEASE WHO DOES NOT HAVE COVID-19 MAY BE A CANDIDATE FOR LUNG TRANSPLANT. SHOULD I STILL REFER MY PATIENT TO A TRANSPLANT CENTER?}

If a patient is a candidate for lung transplant, consideration for referral should proceed. At Cleveland Clinic, as at many other centers throughout the country, we review the available medical information and determine the timeline for evaluation and testing depending on the patient's level of acuity and safety.

\section{WHAT IS HAPPENING WITH PATIENTS WHO ARE WAIT-LISTED FOR LUNG TRANSPLANT?}

Lung transplants are still ongoing at Cleveland Clinic and across the United States. Each individual transplant center is developing criteria to account for how ill a wait-listed patient is, how soon a patient needs to be transplanted, and if it is better for the patient to wait until the pandemic has passed. When making these decisions, we also consider the infectious exposure risks of immunosuppressing the patient in the hospital post-transplant, the infectious exposure risks to surgeons who might have to travel to other parts of the country to procure donor organs, and hospital stewardship policies to ensure there are enough hospital beds and personal protective equipment for nontransplant patients who are admitted to the hospital with COVID-19. To our knowledge, there has been no transmission of COVID-19 from an organ donor to a lung transplant recipient, and this is due in part to the precautions taken by transplant centers and procurement teams around the country.

\section{CONCLUSION}

The care of wait-listed patients and transplant recipients continues, and when these patients are seen in the outpatient or inpatient setting, providers are encouraged to contact the transplant center. The medical literature on COVID-19 is still growing. While there is not yet a report specific to lung transplant, a recent report of 87 heart transplant recipients in Wuhan, China, noted that social distancing coupled with other preventive measures led to a COVID-19 infection rate no higher than that in the general population. ${ }^{2}$ Thus, continued communitybased measures will help protect transplant patients.

\section{REFERENCES}

1. Wang D, B Hu, C Hu, et al. Clinical Characteristics of 138 Hospitalized Patients With 2019 Novel Coronavirus-Infected Pneumonia in Wuhan, China. JAMA 2020 Feb 7.doi: 10.1001/jama.2020.1585 [Epub ahead of print]

2. Ren ZL, Hu R, Wang ZW, et al., Epidemiological and Clinical Characteristics of Heart Transplant Recipients During the 2019 Coronavirus Outbreak in Wuhan, China: A Descriptive Survey Report. Journal of Heart and Lung Transplantation 2020 Mar 25. doi: 10.1016/j. healun.2020.03.008 [Epub ahead of print]

3. Michaels MG, La Hoz RM, Danziger-Isakov L, et al. Coronavirus disease 2019: Implications of emerging infections for transplantation. Am J Transplant. 2020 Feb 24. doi: 10.1111/ajt.15832. [Epub ahead of print] 